\title{
Capturing Conceptual Development through the Embodied-Based Experience in Infinite Sets Comparison
}

\author{
Pakdeeviroch Cherdsak \\ Institute for Innovative Learning, Mahidol University, Thailand, \\ cherdsak.pak@student.mahidol.edu
}

\section{Nokkaew Artorn}

Dr., Corresponding author, Institute for Innovative Learning, Mahidol University, Thailand,artorn.nok@mahidol.edu

\section{Wongkia Wararat}

Dr., Institute for Innovative Learning, Mahidol University, Thailand, wararat.won@mahidol.edu

Designing pedagogical experience to serve as groundwork on which to build an understanding of abstract concepts is a challenging mission for educators. Much research has found that embodied activities could facilitate conceptual metaphor for students to understand such concepts. This study has captured the trajectory of reasoning occurred during the embodied-based experience designed for the infinity concept. The participants, 42 secondary students who had never been exposed to the formal concept of infinity, passed through a designed series of making-sense tasks, so called the Embodied-Based Activity of Infinite Sets Comparison (EBIC). The EBIC intervention was conducted in 2 phases; (i) careful observation of 2 voluntary students, and (ii) integration for 40-classroom practice. The first phase was to examine the students' conceptual development through their utterances, actions and inscriptions. Open-ended questions were used to capture the students' insights and reasoning in the second phase. The qualitative data was collected and mined to associate pairability and Cantor's metaphor. The finding showed the reasoning trajectory of some participants and the shift from improper reasoning to 1-1 correspondence pairing of countably infinite sets. This mathematical thinking shift was a consequence of conceptual embodiment, outlining the analysis of the embodied-based experience to develop abstract reasoning.

Keywords: embodied-based activity, equivalence of sets, infinite set, sets comparison, conceptual development 


\section{INTRODUCTION}

The main obstacle of learning is the lack of experience. This claim is also relevant to the learning and understanding of infinity properties (Denbel, 2014; Tsamir \& Tirosh, 2007; Wijeratne \& Zazkis, 2015). Tall's (2008) mentioned in his transition-in-thinking framework that students lacked infinite experience and hence encountered difficulties when learning in a context of infinite sets. In other words, students had neither experience to perceive, reflect, or sense about the infinite entities in the real world, nor the image of infinite sets comparison. To facilitate the formation of infinity concept, the pedagogical design will have to incorporate meaningful experience along with students' cognitive process monitoring.

This study has aimed to elucidate sequential cognitive reflection by careful capturing of students' reasoning. This is vital as some research has revealed that improper use of reasoning engages an erroneous conclusion in infinite sets comparison (Fischbein et al, 1979; Tsamir \& Tirosh, 2007). The common root of incorrectness encompasses an improper use of reasoning, which is mainly categorized into two types. The first type is overgeneral reasoning, which is overgeneralization of finite-collection methods, relating to inclusion and interval reasoning. Another type is reasoning derived from an inadequate understanding of infinity. Lacking a proper thinking tool or schema to deal with an infinite task, students could generate improper reasoning and hence the thought of infinity as singularity and incomparability (Mamolo \& Zazkis, 2008; Wijeratne \& Zazkis, 2015). Having been introduced to the formal concept of infinite sets comparison, whose traditional approach involves the Cantorian Set Theory, some students still struggled as they inconsistently used several criteria to compare the infinite sets without awareness of a contradiction (Tsamir, 1999). This underpins the fact that content-based approach is insufficient for ones to comprehend abstract ideas. Several studies show that embodied-based activities can support conceptual development (Manches et al., 2010; Nathan \& Walkington, 2017; Petrick et al., 2014). However, the majority of the embodied-based instructional activities are the implementation of introductory mathematics topics, such as proportion, angle, and graph.

To fill this gap and to support students' understanding in the higher-level mathematics concept, we therefore have proposed a novel embodied-based activity, called EmbodiedBased activity of Infinite sets Comparison (EBIC) designed fundamentally based on the Embodied Mathematics Theory (Lakoff \& Núñez, 2000; Núñez, 2005). It is a series of sense-making tasks, where perceptions and actions become linked to promote conceptualization. The anticipated meaningful experience in EBIC is when students embody Pairability concept and develop Cantor's metaphor of the infinite sets comparison. The conceptualization in this aspect emphasizes on a conceptual metaphor instead of a rigorously mathematical proof. Performing EBIC, students' movements echo their learning instantaneously. The investigation of how students understand the abstract term by means of metaphor is carried out with elucidation and classification of students' reasoning associated with each task in the activity. Concretizing the connection between the abstract and the physical movements, this study has not only proposed the 
instructional design and activity but also the trajectory of reasoning, which has shown promise of effective learning underlain by the embodied learning experience.

\section{THEORETICAL BACKGROUND}

\section{Action-Schema-Concept: An Embodied Mathematics Instruction}

Many cognitive science studies reach the conclusion that "abstract concepts are perceptual, being grounded in temporally extended simulations of an external and internal event" (Barsalou, 1999, p.603). This discovery is not only cohesive with the empirical evidence from neuroscience that reveals close relations between action and cognition (Rizzolatti et al., 1996) but also supportive of a belief that mathematics is embodied (Abrahamson \& Lindgren, 2014; Lakoff \& Núñez, 2000). Therefore, some everyday activities can lead to mathematical concept formation as meanings through perceptions and actions are intuitively derived as parts of experiential learning (Abrahamson \& Lindgren, 2014).

The Embodied Mathematics Theory is used to explain how students think of and construct an abstract concept. It provides an elucidation that mathematical concepts can be formed in student' mind through the cognitive mechanisms, which include conceptual metaphor and conceptual blend. A source domain for the cognitive mechanisms is concrete experiences gained through perceptions and actions. This theory claims that mathematics is a product of humans' mind that is comprehended through our brains and bodies. This corresponds to the argument of Lakoff \& Núnez that "human mathematics is not a reflection of a mathematics existing external to human being; it is neither transcendent nor part of the physical universe" (Lakoff \& Núñez, 2000, p.349). As actions and perceptions can lead to concepts, embodied mathematics instruction has been studied in much research, covering mathematics topics, such as addition and partition (Manches et al., 2010), proportion (Abrahamson et al., 2011), angle (Petrick et al., 2014), platonic solid (Parsley \& Soriano, 2009), geometric proof (Nathan \& Walkington, 2017).

\section{Embodied Infinity}

Lakoff and Núñez (2000) proposed that mathematical concepts grounded in physical experience. This comes the curiosity of how to prove concept of infinity where actual infinite experience is hardly experienced in real life. Much research has found that with aids of cognitive mechanisms, conceptual metaphor and conceptual blend in particular, the infinity concept can be embodied in humans' mind (Alibali \& Nathan, 2012; Lakoff \& Núñez, 2000; Núñez, 2005; Singer \& Voica, 2008). A blending process is started with more than one source domains, called input spaces. Construction of a new concept is initiated when inferential mappings link between the input spaces and consequently project onto a target domain, namely a blended space. Therefore, the newly blended concept can contain both the common features from the source domains and a new feature in the blended space (Fauconnier \& Turner, 2002; Mowat \& Davis, 2010). Núñez's research has broadened the conceptualization of infinity by analyzing the Basic Mapping of Infinity or BMI as a double-scope conceptual blending, which involves two input spaces of perfective and imperfective aspects (see Núñez, 2005, p. 1730). The 
process of perfective aspect can be thought of a completed iterative process with an end and a final resultant state. Here, the finiteness is defined. Oppositely, the process of imperfective aspect involves endless iterative process with no end. Here, sense-having of infinity can be formed. The blending of these two aspects deduces an inferential structure organizing "a process with no end and a final resultant state" (Núñez, 2005, p. 1729). This conceptual mechanism is analyzed to echo the conceptual metaphor of infinite sets comparison and its conceptualization. Here, the conceptual metaphor is a Cantor's metaphor, namely Same Number As IS Pairability, described more insightfully in the next section. However, this framework can be theoretically developed to aid the learning of infinite comparison.

\section{Pairability and Cantor's Metaphor}

In the finite context, the Same Number As concept is employed to verify that a collection $A$ and a collection $C$ are of the same cardinal number. They are so if after taking away each element of $A$ and a corresponding element in $C$, none of the element left over in both collections, see Figure 1(a). The More Than concept is used to substantiate that $B$ has more elements than $C$. It does so, if after taking away each element of $C$ and a corresponding element in $B$, there is at least one element left in $B$, see Figure 1(b) (Núñez, 2005). However, these ordinary concepts seem insufficient to infer infinite comparison as the infinite process involves no ending state. Thus, figuring out the residual of taking away process is perhaps problematic.

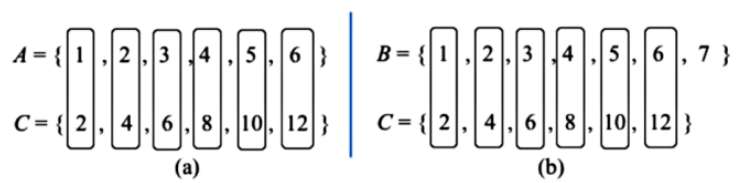

Figure 1

(a) The use of the Same Number As, and (b) More Than Concept in Finite Comparison

The lack of a proper concept in infinite comparison results in overgeneralization of finite context as intuitive reasoning. Accordingly, students make an incorrect conclusion. As shown in Figure 2(a), in comparing the set of natural numbers and the set of positive even numbers, the inclusive reasoning and the More Than concept are intuitively used. This falsely leads to the conclusion the set of natural numbers has more elements than the set of positive even numbers. Countering this intuitive consideration, George Cantor (1845-1918) proposed a new concept called Pairability in replacement of Same Number As and More Than concept. Any two sets meet Pairability if they can be put into 1-1 correspondence. Thinking back to the previous example, for every $n$ in the natural numbers, we can put $e=2 n$ where $e$ is an element of the set of positive even numbers. Corresponding to 1-1 relation, both sets satisfy Pairability and have the same number of elements, see Figure 2(b). In this sense, Pairability provides reconceptualization for the Same Number As concept. The framework of conceptual metaphor is applied to make the final resultant state of the infinite process sensible. This conceptual metaphor is called Same Number as IS Pairability (Lakoff \& Núñez, 2000). Furthermore, Pairability concept is effectively practical in both finite and infinite 
entities (Tsamir \& Dreyfus, 2002). It is an extraordinary concept that unnaturally existed in human (Lakoff \& Núñez, 2000; Núñez, 2005).

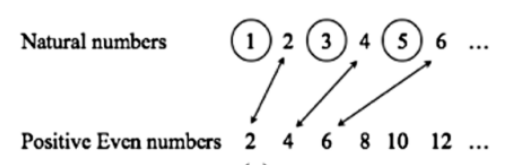

(a)

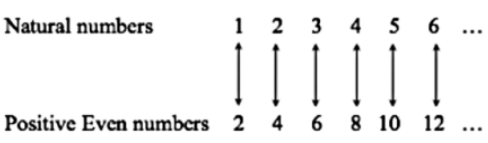

(b)

Figure 2

(a) The use of the More Than and (b) The use of Pairability within Infinite Context

\section{THE STUDY}

As mentioned, provision of experience for students to grow concepts is critical. In particular, experience in comparing infinite entities is difficult to stumble upon in real life. In this section, the activity called Embodied-Based activity of Infinite sets Comparison (EBIC) is presented. This offers the experience that would overcome the difficulty in understanding infinity. With the careful methodology, we can also study the cognitive process of conceptualization.

\section{Embodied-Based activity of Infinite sets Comparison or EBIC}

To design embodied-based activity, Abrahamson and Lindgren (2014) suggested considering activities, materials, and facilitation. In other words, the learning environment and physical interaction should be designed to promote the emergence of conceptual reasoning. Furthermore, the appropriate educational materials are suggested in teaching mathematics (Kul et al., 2018). This section presents a set of activities designed to promote conceptualization of Pairability concept and Cantor's metaphor.

We designed the EBIC activity to serve the purposes, which are 1) to evoke the ordinary concepts, Same Number As, and More than, 2) to develop Pairability idea and to conceptualize the finite comparison using the conceptual metaphor with the Same Number As IS Pairability scheme, 3) to facilitate students to conceptualize the infinite comparison using the conceptual metaphor with the Same Number As IS Pairability scheme which integrates the designed mechanism of BMI framework, and 4) to underpin the ideas gained from participating in the activity by the problem application. The EBIC activity series began within the finite context and gradually moved to the infinite context as shown in Table 1. In the process of activity, no formal definition related to Cantorian Set Theory was prompted to students. Due to the limitation of space, the details of the tasks will be explained along with the results in the next section. 
Table 1

The Embodied-Based Activity of Infinite Sets Comparison (EBIC)

\begin{tabular}{|c|c|c|c|c|}
\hline Part & Embodiment & Key tasks & Task characteristics & Contexts \\
\hline I & Same Number As & $\begin{array}{l}\text { - Compare sizes of the two given } \\
\text { collections of physical objects without } \\
\text { counting }\end{array}$ & $\begin{array}{l}\text { - Finitely small numbers } \\
\text { of objects - Concrete and } \\
\text { visible objects }\end{array}$ & \\
\hline II & $\begin{array}{l}\text { Same Number As IS } \\
\text { Pairability (Blending } \\
\text { Same Number As and } \\
1-1 \text { correspondence) }\end{array}$ & $\begin{array}{l}\text { - Create the two finite sets from a } \\
\text { relation discovered from the several } \\
\text { representations including geometric and } \\
\text { numeric-vertical representation } \\
\text { - Compare the size of the two sets }\end{array}$ & $\begin{array}{l}\text { - Finitely large numbers } \\
\text { of elements } \\
\text { - Symbolic and invisible } \\
\text { elements }\end{array}$ & Finite \\
\hline III & $\begin{array}{l}\text { Same Number As IS } \\
\text { Pairability (Blending } \\
\text { Imperfective and }\end{array}$ & $\begin{array}{l}\text { - Create two countably infinite sets } \\
\text { following the relation prompted and take } \\
\text { away each pair of members }\end{array}$ & $\begin{array}{l}\text { - Infinitely many } \\
\text { numbers of elements }\end{array}$ & \\
\hline IV & $\begin{array}{l}\text { Perfective aspect) } \\
\text { Same Number As IS } \\
\text { Pairability }\end{array}$ & $\begin{array}{l}\text { - Compare the size of the two sets } \\
\text { - Compare sizes of two given countably } \\
\text { infinite sets } \\
\text { - Categorize the infinite sets into either } \\
\text { countable set or uncountable set }\end{array}$ & $\begin{array}{l}\text { - Infinitely many } \\
\text { numbers of elements }\end{array}$ & Infinite \\
\hline
\end{tabular}

\section{METHOD}

This study was mainly focused on the engagement of EBIC activity to students' learning and conceptualization of the infinite sets comparison by capturing the path of students' reasoning. The qualitative approach was adopted to investigate the trajectory of reasoning during series of sense-making tasks for infinite sets comparison. The performance of students undergoing EBIC activity included their utterances, actions, and inscriptions. These shall be interpreted into changes in reasoning toward finite and infinite sets comparison. Tracking embodied learning, we meticulously observed students in real scenario and from video recording. The open-ended questions were designed purposely and were used to capture the changes in reasoning and henceforth conceptualization.

\section{Participants}

There were two phases of study. In the first phase, the careful observation, a purposive sampling was used to recruit two secondary students, James and Oak as the pseudonym, in the observation. They were studying in grade 11 of the normal program in a regular public school administered by Thai educational policy. Both of them had average mathematics competency. James and Oak learned the fundamental of sets. However, they have never learned the concept of comparing the cardinality of infinite sets. In the second phase, the 40-classroom practice, the purposive sampling was administered again to recruit other 40 voluntary $11^{\text {th }}$ graders, both boys and girls 16-17 years of age, into the intervention. The characteristics of them, 40 students, were identical to James and Oak.

\section{Procedure and Data Collection}

We created the EBIC activity fundamentally based on the Embodied Mathematics Theory for infinite sets comparison. It aimed to promote students' conceptualization 
following Same Number As IS Pairability scheme which would facilitate proper reasoning. The details of the activity follow Table 1 and are delineated in Findings section.

For the first phase, a careful observation of two voluntary students, the EBIC activity was conducted following the four parts of embodiments, shown in Table 1. While the participants underwent the EBIC activity, video recorded their actions, conversation they had with the researcher who acted as a teacher and other researchers, a team of three, as the observers. Both participants spent on average an hour and a half to complete each embodiment. Their inscriptions and scratch during the activity were kept to be discussed. In this phase, we looked profoundly into how the EBIC activity promoted participants' proper reasoning. Therefore, monitoring movements and path of reasoning of the two participants, James and Oak, were critical in this phase.

For the second phase, the 40-classroom practice, we studied whether the EBIC activity influenced the change in reasoning from intuitive reasoning to 1-1 correspondence pairing in classroom setting. For the 40 -classroom practice, we purposively recruited 40 upper secondary students into the classroom practice. The EBIC activity was implemented for the groups of four participants performing the tasks in sequence together. Note that the tasks of EBIC activity were the same as for the first phase. In this phase, we divided the procedure into 3 steps; pre-intervention, intervention, and postintervention. All interventions were conducted by the researchers, a team of three when one taught, the other ones observed. In the pre-intervention step, participants answered the open-ended questions pertaining to infinite sets comparison. In this step, six questions were presented in numeric-horizontal form situated from task to task. Five of them involved only countably infinite sets. The other one was related to the comparison between countably infinite set and an uncountable set (Figure 3). Questions 1 and 2 were designed intentionally to provoke participants to use inclusive reasoning. Question 3 was designed to trigger the use of interval reasoning. Challenging further, the set of natural numbers was removed in question 4 and 5 and replaced by the sets of sequential integers. And finally, the real number set, denoted by $R$, was presented in question 6. Participants were asked to determine cardinal equivalence and give reasons to support their answers.

\begin{tabular}{|llll} 
Question 1: $A=\{1,2,3,4,5, \ldots\}$ & $\mathrm{B}=\{1,3,5,7,9, \ldots\}$ & Question 2: $\mathrm{A}=\{1,2,3,4,5, \ldots\} \quad \mathrm{B}=\{5,6,7,8,9, \ldots\}$ \\
Question 3: $\mathrm{A}=\{1,2,3,4,5, \ldots\}$ & $\mathrm{B}=\left\{0, \frac{1}{2}, \frac{2}{3}, \frac{3}{4}, \frac{4}{5}, \ldots\right\}$ & Question 4: $\mathrm{A}=\{-1,-2,-3,-4, \ldots\} \quad \mathrm{B}=\{3,6,9,12, \ldots\}$ \\
Question 5: $\mathrm{A}=\{1,4,9,16, \ldots\}$ & $\mathrm{B}=\{4,8,12,16, \ldots\}$ & Question 6: $\mathrm{A}=\{1,2,3,4, \ldots\} \mathrm{B}=R$ where R denotes set of real numbers
\end{tabular}

Figure 3

The Infinite Sets Comparison Questions in the Open-Ended Questions

Next, the intervention step was where we introduced the designed EBIC activity consisting of four embodiments, shown in Table 1. For the second phase, 40-student classroom, we organized each embodiment in four daily 2-hour classes. The researcher conducted the EBIC activity class as a teacher, the others observed the class with video recording to collect participants' actions throughout the process of intervention. Again, the tasks of EBIC activity were sequentially implemented the same as for the first phase. With concerns of research methodology and embodied perspective, an encouragement 
of individual's experience with the tangible manipulative was emphasized by individual questioning and group discussion throughout an intervention. Lastly, for the postintervention step, the open-ended questions, identical to the pre-intervention, were applied again. The reasoning of participants according to infinite sets comparison was captured by the open-ended questions for both before and after EBIC activity intervention.

\section{Data Analysis}

To track the development of participants' reasoning, the video of two voluntary participants performing EBIC tasks was segmented and analyzed inductively. The utterances, actions, and inscriptions of the two voluntary participants were examined together for utmost cogency in the interpretation (Arzarello et al., 2009; Chahine, 2013; Edward \& Robutti, 2014). A consensus among the researchers about participants' responses was also obeyed to establish consistency and the credibility of the conclusions.

Table 2

The Reasoning, the Descriptions of Reasoning, and Types of Reasoning

\begin{tabular}{lll}
\hline Reasoning & Descriptions & Types of reasoning \\
\hline Pairing & Every element can be put into 1-1 correspondence & Proper reasoning \\
\multirow{2}{*}{ Inclusion } & $\begin{array}{l}\text { A proper subset of given contains fewer elements than the set } \\
\text { itself } \\
\text { A bounded set contains fewer elements than an unbounded set } \\
\text { A bounded set contains fewer elements than an unbounded set }\end{array}$ & $\begin{array}{l}\text { overgeneralized from finite } \\
\text { collection methods }\end{array}$ \\
\hline Interval & $\begin{array}{l}\text { A set where has longer consecutive distance contains fewer } \\
\text { elements }\end{array}$ & $\begin{array}{l}\text { overgeneralized from finite } \\
\text { collection method }\end{array}$ \\
\hline Singularity & All infinite sets are equal & $\begin{array}{l}\text { Inadequate understanding } \\
\text { of infinity }\end{array}$ \\
\hline Incomparability & Infinite sets are incomparable & $\begin{array}{l}\text { Inadequate understanding } \\
\text { of infinity }\end{array}$ \\
\hline
\end{tabular}

In the classroom practice, the reasoning of the participants in the open-ended questions was deductively categorized into the thematized reasoning types: pairing, inclusive, interval, singular, and incomparable reasoning (Fischbein et al., 1979; Tirosh, 2002). The description of reasoning and types of reasoning for infinite sets comparison are shown in Table 2. To establish validity, the participants' responses were deliberately interpreted and categorized according to the consensus among three researchers (Denzin \& Lincoln, 2005). To draw a legitimate conclusion, we aligned data from the observation and from the open-ended questions. This led to the promising results of the EBIC activity to aid infinite comparison concept.

\section{FINDINGS}

This section presents the findings of the two phases. However, first, we will delineate the results from careful observation. This gives comprehensive utterances, actions, and inscriptions of the two volunteers, James and Oak. Capturing four critical actions observed in the EBIC activity would benefit the interpretation of data in the 40classroom practice phase or conclusively general cases. 


\section{The Developmental Trajectory of Reasoning in Infinite Sets Comparison}

Critical Action 1: Embodied Pairing Concept

For finite context, the participants were shown the images of LEGO pieces in a flash then were asked "Are quantity of green and red LEGO pieces equal?" and "How would you justify your answer?". Both observed participants used counting.

James: Count to find how many pieces of this color and how many pieces of the other color.

Oak: Count and quantify them, then, compare the quantity.

Next, the participants were further asked to demonstrate the strategies to compare the numbers of the two collections without counting. Two sets of candies were provided as physical manipulatives. The arrangement of candies demonstrated by the participants was shown in Figure 4.

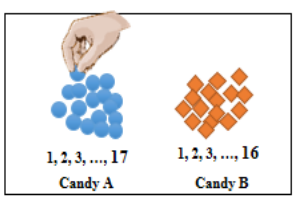

(a)

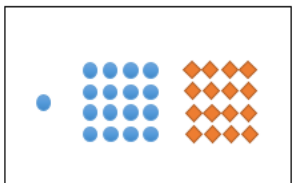

(b)

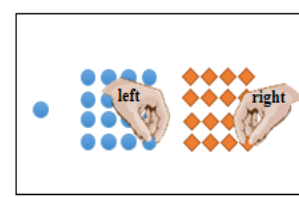

(c)

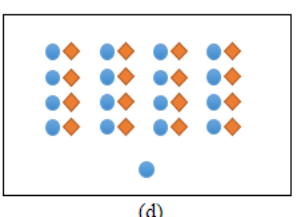

(d)

Figure 4

The Graphics Illustrating James' Thinking

Firstly, James grouped the orange and the blue candies, arranged each group to have similar square shape, and left the residual apart, as in Figure 4(b). After that, he altered the form of arrangement to justify the inequality. He possibly thought about more effective presentation to grasp whether the quantities of the two candies are equal or not. He grabbed the candies one by one rearranged them in pairs of blue and orange as shown in Figure 4(c) and 4(d).

James: The number of each type of candy is different. When they were paired and there was the residual, it means that the quantities of the two candies are not equal. But after pairing if no residual, then the quantities of the two candies are equal.

For the tangible comparing, James used 'residual' as an artifact to draw a conclusion instead of the count of the candies. Oak expressed his solution in the similar fashion.

Oak: There is another way to show that each type of candy can be paired. We look at the size of the arrangement. Both orange and blue candies can be arranged in $4 \times 4$ shape. But the blue has one left. It is similar to pairing one blue and one orange with one blue left unpaired.

Instructor: Is the previous method [Figure 4(b)] considered as the pairing?

Oak: Yes, ...group pairing.

Expressed explicitly in actions, utterances, and inscriptions, the More than concept was utilized in making a conclusion of the comparison. In addition, we could observe the combination of the More than and the pairing to re-conceptualize 'counting', as Oak 
mentioned. The counting method was re-described in pairing image, as they called 'group pairing' (Figure 4(b)).

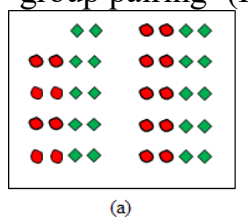

(a)

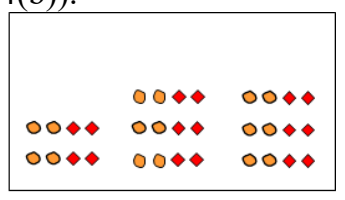

(b)

Figure 5

The Pairing Arrangement Comparing the Quantities of Candies of (a) Oak and (b) James

Once emerged, the pairing image was used consistently for the later tasks. Besides, they recognized themselves that they could compare without knowing the total numbers of the compared collections. Thus, 'residual' became an attention that both Oak and James embraced. It was noticeable in the arrangements that Oak and James performed the alternative way of paring shown in Figure 5(a) and 5(b) respectively.

Oak: They are not equal. After pairing two reds with two greens, I found the residuals which are 2 green candies, no red candies left to be paired.

James went further in his reasoning as he mentioned the relation in which the number of red and orange candies had to be equal.

James: They are equal. I grouped 2 reds and 2 oranges as one set. When all candies are grouped, we can tell if they are equal without knowing the number of red and orange candies. It is because in one set, the number of red and orange candies are equal. So, no residual means that we can make sets that red and orange are equal.

Moreover, he also explained that;

James: Counting and pairing give us the same result. When we use counting method. ... For example, the quantity of the first collection is 5 and the second one is also 5, then they are equal. Likewise, if there is no residual when we pair up something, as each type has 5 things, it means they are equal.

James re-conceptualized the counting method as a conditional pairing, namely equal pair, which did not require knowing the quantities but residual. This concept was formed in Oak and clearly observed when he explained the pairing method in the different context.

Instructor: Suppose we count the number of two collections and get 120 and 121 respectively. What can we say if we use the pairing method instead?

Oak: Remainder is 1. I decomposed 120 to $1+1+1+\ldots, 120$ terms. In the same way for 121 , I have $1+1+1+\ldots+1,121$ terms. After that, I paired each number ' 1 ', one from 120 and one from 121. Finally, there is a number ' 1 ' of 121 left.

With finite context, the tangible manipulatives and questioning fruitfully facilitated the participants to re-conceptualize counting as a conditional pairing in finite sets comparison. The reasoning path of Oak and James revealed the changes when the amount of candies was no longer necessary to them to compare two sets. Counting method was then removed and replaced by pairing method and checking the residual. It 
is an evidence of the emergence and the use of the Same Number As and the More than image in reasoning.

Critical Action 2: Conceptualizing 1-1 correspondence properties

After the participants constructed images of pairing, there came the second part of the activity where the participants were brought from the concrete realm to more mathematical realm. They were expected to apply the image of pairing to the new context. The new task was designed to support the extension of pairing. The geometric representation well aided the 1-1 correspondence comprehension, which is essential for mathematical comparison (Tsamir \& Tirosh, 1999).

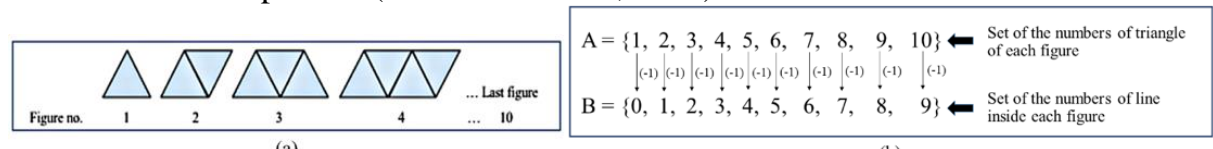

Figure 6

(a) The Geometrical Representation; (b) The Example of the Solution of the Participant

In the first task, there were 10-term geometrical pattern as in Figure 6(a). The participants constructed two sets of numbers representing the geometrical pattern, as in Figure 6(b). Then, the participants were challenged by the question, what if we have 100 -term or 1,000-term triangle patterns, how would we find 1-1 relation?

Oak: Yes, ... because each element of set A has one corresponding element of set $B$.... if we increase the members of set A following the relation, then the members of set $B$ would be increased parallelly and no member in set $B$ is repeatedly constructed.

James: I think the members of set $B$ are generated from the members of set A... A grows its member, $B$ grows in correspondence. The increment of the number of elements in set $A$ and set $B$ is a constant. If both sets have infinite members, they are still pairable.

Both participants viewed 'pair' as a relation of elements. Oak also noticed the characteristics of member generation regarding to the relation. He said, "no member in set $B$ would be repeatedly constructed". He observed 1-1 correspondence. More interestingly, James seemed to extend his idea from finite to infinite comparison. Valuable inference from this task was where geometric representation could engage the 1-1 correspondence pairing.

Next, the representation was changed from geometric to numeric representation. The participants were assigned to work individually to compare the finite sets presented in the numeric form. They not only paired the members between two given sets, but they also specified the corresponding relation. For example, Oak identified the relation between set $A$ and set $B$, which was incrementing by 2 , as shown in Figure 7(a). He created 1-1 relation - one member of $A$ corresponds to one member of $B$. James also showed the same idea. 
Even more challenging to discover $1-1$ relationship, $C=\{1,2,3, \ldots, 115\}$ and $D=\{1$, $4,9, \ldots, 13225\}$ were given, where the symbol '...' was used to represent the hidden elements. Apparently, the participants could not see if the numbers of the element in both sets were equal.

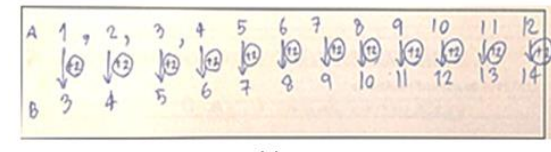

(a)

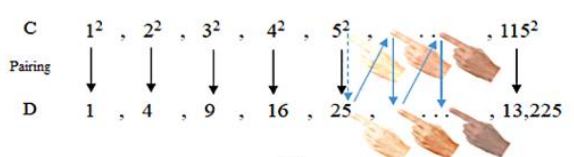

(b)

Figure 7

Students' Reasoning for Finite Sets Comparison Problems, (a) Small and (b) Large Finite Sets

Oak and James found the same relation, which was squaring that linked each member of $C$ to $D$. Oak explained the idea of pairing through his hand motions and utterance, as portrayed in Figure 7(b). Then, the participants were asked to summarize the comparison concept in their own words.

Instructor: From this activity, could you please conclude the characteristics of the relation that can justify the equivalence of any two sets.

Oak: One element of set A constructs one element of set B. Or, the elements of set $A$ can create the elements of set $B$. Therefore, the number of A grows, the number of $B$ grows.

James: The two sets are equal if and only if there is a relation used to construct members one by one. It is like one to one relation.

In summary, working in geometric representation gave the guidance to a 1-1 correspondence concept. The relation with such characteristics could help justify equal size of two sets. In addition, after removing geometric patterns, the concept of 1-1 correspondence in numeric representation could be formed. Interestingly, the use of geometric representation could be the starting point to observe 1-1 relation, which geared the participants to achieve 1-1 correspondence for numeric patterns representing finite or infinite sets comparison.

Critical Action 3: Same Number As IS Pairability, a conceptual blending for finite context

After geometric and numeric representation, the participants achieved 1-1 correspondence and recognized the significant role of 1-1 correspondence as a criterion to justify equivalency of sets. The participants used this idea in comparing sets, shown in their utterances as follows.

James: We have to consider whether there is the one to one relation [1-1 correspondence] between set $A$ and set $B$ or not.

Oak: For me, I think, ..., we can count and then compare, yet if the numbers of two sets are too many the finding relation is more suitable than counting. 
We began the next task by asking "what is the result of taking away every pair?" The answer of the participants was "empty sets". The excerpts below from James and Oak showed their explanation indicating that conceptual metaphor of removing paired members cultivated in their thoughts.

Oak: If every member has been paired following the relation [1-1 correspondence], then we take away every paired member. The result is that in each set, there is no remaining member that would have no pair.

James: Because the latter set is generated from the former set following the relation [1-1 correspondence]. So, if each pair was taken away until it's done,... since both sets are into the relation, eventually, both sets are the equally empty sets.

Both participants mentioned empty sets or no remainder with similar reason that both sets were paired in 1-1 relation. Then, they were introduced the formal definition of 1-1 correspondence. Then, we posed more conceptual question to them, "what does it mean if the two sets can be put into 1-1 correspondence?" Both of them replied that the two sets were equivalent, their members were paired 1-1.

In summary, the series of tasks in this part successfully promoted the conceptual blending of the Same Number As concept and Pairability, provoked in part I and II respectively. The critical evidence was the meaning in action, saying if every member of the two (finite) sets can be put into 1-1 correspondence, they are equivalent set. The participants conceptualized Same Number As IS Pairability for the finite context. This unveiled the metaphorical blending process referring to BMI cognitive mechanism.

Critical Action 4: Same Number As IS Pairability, a conceptual blending for infinite context

Once Pairability and Same Number As IS Pairability emerged, the transformation of these concepts from finite context to infinite context was conceivable. The participant experienced the perfective aspect and the final resultant state, "no remainders after taking away every pair'. The BMI cognitive mechanism that was embedded in the previous part would serve a good foundation to build on imperfective aspects involving endless iterative process. The first task in this part was to create two infinite sets. The participants randomly picked a relation, which generated numeric pairs of $n$ and $k$. They did not know at first whether the picked relation was 1-1 correspondence or not. The manner of the task was that before generating the new pair of $n$ and $k$, the participants had to cross out the previous pair. Here, the 'crossing-out' action hence was metaphorical representation. Then, the participants completed the first task as shown in Figure 8 .

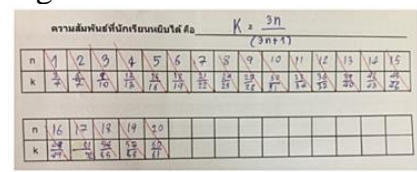

(a)

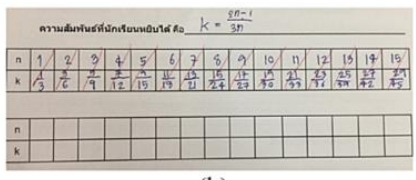

(b)

Figure 8

The Inscriptions of (a) James's and (b) Oak's Responses in the Table of $n$ and $k$ 
The participants realized that the picked relations were 1-1 correspondence. They constructed pairs of $n$ and $k$, one in the set $A$ and set $B$ respectively. However, they did not fill up the table, as shown in Figure 8(a) and 8(b). Then we asked if they knew that these set were finite or infinite.

Oak: Infinite sets [Immediately replied].

James: ...Yes [infinite sets].

Instructor: What is your reason?

James: $n$ is any natural number which can be increased inexhaustibly. So $k$ was constructed by $n$, like one by one, $k$ is unlimited as well. Therefore, both sets are infinite sets.

Next task was the infinite sets comparison. The dialogues shown below are the dialogue of participants' reasoning.

Instructor: Why were you sure that both infinite sets were equivalent?

James: Both sets have the same number of members because the members of set $B$ were created by the members of set A like... one by one.

Oak: There is no member left after taking away every paired member.

Instructor: Why? As you mentioned earlier, you don't know the last member.

Oak: Because both sets have relation together that is 1-1 correspondence ... when a member of one set is increased, then it will create a corresponding member according to 1-1 relation for the another set. So, every pair has to be taken away simultaneously as well.

James: Like I said, the key is 1-1 correspondence, ...we continuously create the corresponding pairs. So, finally, all members of the latter set were created by the members of the former set.

Instructor: Even though, both sets are infinite?

James: Definitely, they are the infinitely pairable sets.

The participants symbolically thought of the crossing-out action as the taking-away action. In this way, this clearly showed the taking away of both sets yielded the empty sets. Extending to infiniteness, both participants reasoned that the two constructed infinite sets were equal because every member of both sets could be put into 1-1 correspondence.

In summary, the participants fully adopted 1-1 correspondence to justify equivalency of infinite sets. This image of pair removal constructed in part II gave embodied learning to conclude 'final resultant statement' of 'iterative endless process'. As the result, the double-scope conceptual blending by two input spaces, perfective and imperfective process, offers the advantage in blending and transforming the metaphor, Same Number As IS Pairability, from finite context to infinite context.

The careful observation phase gave a meaningful critical action for each part of EBIC activity. This would further serve as set of benchmarks in conceptualization for infinite 
sets comparison. Table 3 shows the developmental trajectory of reasoning that the participants expressed through the EBIC activity.

Table 3

The Developmental Trajectory of Reasoning of the Participants through EBIC Sctivity

\begin{tabular}{|c|c|c|c|c|}
\hline Critical events of developmental & Context & Spot & \multicolumn{2}{|c|}{ Focus in reasoning } \\
\hline Counting & Finite & Part I & Total numbers & - \\
\hline $\begin{array}{l}\text { Pairing } \\
\text { (Same Number AS and More Than) }\end{array}$ & Finite & Part I & Residual & $\begin{array}{l}\text { Relation with condition } \\
\text { (identical group) }\end{array}$ \\
\hline $\begin{array}{l}\text { Pairing and 1-1 correspondence } \\
\text { (Same Number AS and More Than) }\end{array}$ & Finite & Part II & Residual & $\begin{array}{l}1-1 \text { correspondence } \\
\text { relation }\end{array}$ \\
\hline $\begin{array}{l}\text { Pairing and 1-1 correspondence } \\
\text { (Same Number AS and More Than) }\end{array}$ & Infinite & Part III & Residual & $\begin{array}{l}1-1 \text { correspondence } \\
\text { relation }\end{array}$ \\
\hline
\end{tabular}

The achievement shown from Oak and James' cases drove us to classroom practice. Referring to the path of reasoning and critical actions from phase I, the six open-ended questions were deliberately designed according to BMI cognitive mechanism. The 3step procedure produced the data in the form of participants' physical actions, conversation and writing that showed their reasoning corresponding to the designed tasks and questions, which were categorized and yielded the following findings.

\section{The Changes in Reasoning, the Responses in the Open-Ended Questions}

The percentage of participants' responses to the open-ended questions in preintervention and post-intervention are shown in Table 4. In the pre-intervention step, the use of improper reasoning was pervasive. Only one student utilized pairing reasoning in comparison to question 3 to 5 . No one used pairing reasoning for question 1, 2 and 6 . The major types of reasoning found in pre-intervention step were the singular and inclusive reasoning. In post-intervention step, there was a dramatic change. For question 1 to $5,80 \%$ of participants, or more, exhibited pairing reasoning. However, in question 6 , only a quarter of participants used pairing reasoning, while about $40 \%$ of participants used inclusive reasoning

Table 4

The Percentage of Participants' Responses in the Open-Ended Questions in Pre- and Post-Intervention Step $(n=40)$

\begin{tabular}{cllllllllll}
\hline Q. & \multicolumn{2}{c}{$\begin{array}{l}\text { Pre-intervention step } \\
\text { Pairing }\end{array}$} & Singular & Inclusive & Interval & Incomparable & $\begin{array}{l}\text { Post-intervention step } \\
\text { Pairing } \\
\text { Singular }\end{array}$ & Inclusive & Interval & $\begin{array}{l}\text { Incompar } \\
\text { able }\end{array}$ \\
\hline 1 & 0.0 & 37.5 & $50.0^{*}$ & 0.0 & 12.5 & $87.5^{*}$ & 5.0 & 5.0 & 0.0 & 2.5 \\
2 & 0.0 & 40.0 & $45.0^{*}$ & 2.5 & 12.5 & $85.0^{*}$ & 0.0 & 7.5 & 0.0 & 7.5 \\
3 & 2.5 & $45.0^{*}$ & 7.5 & 15.0 & 30.0 & $80.0^{*}$ & 2.5 & 2.5 & 2.5 & 12.5 \\
4 & 2.5 & $45.0^{*}$ & 2.5 & 27.5 & 22.5 & $87.5^{*}$ & 0.0 & 2.5 & 0.0 & 10.0 \\
5 & 2.5 & $45.0^{*}$ & 2.5 & 17.5 & 32.5 & $85.0^{*}$ & 0.0 & 0.0 & 0.0 & 15.0 \\
6 & 0.0 & 27.5 & $47.5^{*}$ & 0.0 & 25.0 & 25.0 & 15.0 & $40.0 *$ & 0.0 & 20.0 \\
\hline
\end{tabular}

\section{Failure of using pairing}

Even though a number of participants used pairing reasoning in the post-intervention step, not every use of pairing reasoning was successful. Evidence of this was the responses to the questions. It showed that not every use of pairing reasoning led to the 
correct comparison of infinite set. Table 5 shows the percentage of correct and incorrect answers of the participants. The percentage of successful use of pairing reasoning depends on the questions. In question 2, all participants who used pairing reasoning answered correctly. Whereas only $35 \%$ from $85 \%$ who chose to use pairing reasoning got the question 5 correct. An in-depth analysis upon various evidence sources, inscriptions, recorded motions and conversation, revealed that they adopted embodied scheme in Pairability but could not figure out 1-1 correspondence. Similar finding in question 6 , only $15 \%$ from $25 \%$ who applied pairing reasoning got the question correct. Whereas, everyone who performed inclusive reasoning got it correctly. This steered to the interest of what could be the factors of successful use of Pairability.

Table 5

The Percentage of Participants' Responses to the Open-Ended Questions in PostIntervention Step $(n=40)$

\begin{tabular}{|c|c|c|c|c|c|c|c|c|c|}
\hline \multirow{3}{*}{ Q. } & \multicolumn{9}{|c|}{ Types of reasoning } \\
\hline & \multicolumn{2}{|c|}{ Pairing } & \multicolumn{2}{|c|}{ Singular } & \multicolumn{2}{|c|}{ Inclusive } & \multicolumn{2}{|c|}{ Interval } & \multirow{2}{*}{$\begin{array}{l}\text { Incomparable } \\
\text { ICR }\end{array}$} \\
\hline & $\mathrm{CR}$ & ICR & $\mathrm{CR}$ & ICR & $\mathrm{CR}$ & ICR & $\mathrm{CR}$ & ICR & \\
\hline 1 & $72.5 *$ & 15.0 & 5.0 & 0.0 & 0.0 & 5.0 & 0.0 & 0.0 & 2.5 \\
\hline 2 & $85.0 *$ & 0.0 & 0.0 & 0.0 & 0.0 & 7.5 & 0.0 & 0.0 & 7.5 \\
\hline 3 & $57.5 *$ & 22.5 & 2.5 & 0.0 & 0.0 & 2.5 & 0.0 & 2.5 & 12.5 \\
\hline 4 & $60.0 *$ & 27.5 & 0.0 & 0.0 & 0.0 & 2.5 & 0.0 & 0.0 & 10.0 \\
\hline 5 & $35.0 *$ & 50.0 & 0.0 & 0.0 & 0.0 & 0.0 & 0.0 & 0.0 & 15.0 \\
\hline 6 & 15.0 & 10.0 & 0.0 & 15.0 & $40.0 *$ & 0.0 & 0.0 & 0.0 & 20.0 \\
\hline
\end{tabular}

\section{DISCUSSION AND CONCLUSIONS}

The development of conceptual understanding is a vital element for mathematics teaching. The embodied-based activity in this study was designed with aims to conceptualize countably infinite sets comparison. The empirical data of two phases in the study design gave significant cognitive benchmarks in embodied learning. In this section, we referred all participants as students. The important remark in this embodied learning process is that no formal mathematical definition was provided to students. Students were expected to utilize and extend their ordinary concept of counting to construct Cantor-like metaphor. With proper metaphor and reasoning, students could generate their definition based on their embodied-based experience.

Working on the topic of infinity, assumed to be imponderable to most students, we confirmed this by the pre-intervention data that infinite set comparison is hardly embodied through daily-life experience (Lakoff \& Núñez, 2000; Núñez, 2005). Regarding to the responses to the open-ended questions and the observation, although students fluently used pairing reasoning in the finite problems, they struggled to form pairing images when dealing with infinite comparison problems. Therefore, the percentage of students who used pairing reasoning to tackle an infinite set comparison is small $(\leq 2.5 \%)$ in the pre-intervention step. Undergoing the embodied activity, the students have shown path of their reasoning. They were geared to construct proper images and reasons for infinite sets comparison. The interesting path of reasoning showed the changes in focus from a quantity to a residual or a remainder when comparing two sets. The impact of the embodiment is relevant to this growth of 
reasoning as the Cantor's metaphor Same Number As IS Pairability being generated. The proper reasoning for infinite set comparison hence has the groundwork to build on. Performing in the post-intervention, students utilized the Pairability concept at a high rate, resembling the studies of Tsamir (1999) and Tirosh (2002) in which university students learned the enrichment course and the learning unit, respectively. Identifying 11 correspondence following the realization of Pairability is the cognitive benchmark showing how students gradually connect the embodied-based experience to a mathematical idea.

In Thai mathematics context, the infinite sets comparison is not in mathematics curriculum for a secondary student. It is in the university level. This is a rationale that the comparative study between the learning with the EBIC activity and the traditional teaching could not be accomplished. Therefore, this study aimed to explore whether students in the secondary level (16-17 years old) can construct and develop a thinking process, reasoning, related to infinite sets comparison concept which is a higher mathematical concept. Fascinatingly, the findings of this study suggest that the EBIC activity can be implemented in class for secondary students to achieve 1-1 pairing reasoning and concept of infinite comparison without formal introduction of the concept definition in which the Cantorian Set Theory was introduced to provide the reasoning tools for students. This implies that students in the lower level are able to develop a higher mathematical idea through a well-designed activity. This fruitful implication corresponds to the previous studies that the appropriate actions, materials, and facilities should be considered harmonizing with the experience which is the source domain in the cognitive mechanism (Abrahamson \& Lindgren, 2014; Kul et al., 2018; Lakoff \& Núñez, 2000).

The fact that reasoning involves existing knowledge and experience is inevitable. In terms of correctness, some prior knowledge in mathematics, such as the concept of composite function, is found to affect the correctness of students' answers. Presenting in the data and observation, we investigated students who used a pairing method in their reasoning. However, some students gave incorrect conclusions of the comparison. Upon the analysis, we found that they struggled to identify the 1-1 correspondence in the infinite sets comparison particularly when the comparing sets were not the set of natural numbers. Moreover, students revealed difficulty in associating countable with the uncountable sets. For example, only a few students used pairing reasoning and most of them showed incorrect and inconsistent reasoning in question 6. This has led to the conjecture about prior knowledge and students' experience could be the factors. Because students gain experience from their intuition and previous instructions, the cognitive structures accommodate the familiar information, similar problems or kinds of representation. This finding corresponds to the study of Petrick et al. (2014) mentioning about the students failing to make the connection between representations in the embodied-based learning.

However, this study only involved 42 secondary students ( 2 students for careful observation and 40 students for classroom practice). Thus, various cases of students' embodied-based learning can be further explored to obtain wider impact and thoroughly understand EBIC activity intervening cognitive process of Pairability conceptualization. 
Nonetheless, the findings of this study gave us insight into how students approached advanced mathematical concepts via an alternative embodied-based instruction. The open-ended questions applied before and after gave comparable results. Some performed proper reasoning derived from embodied-based activity but failed to answer the questions correctly. This phenomenon can be a result of insufficient knowledge backgrounds, in particular, some that are relevant to mathematical concepts, such as the composite function.

As for future work, in-depth study with a larger numbers and wider range of participants is recommended. Besides, the cause of inconsistent reasoning, inconsistent use of the embodied metaphor could be further investigated. The entailing research series perhaps involves how students connect concepts to embodied-based experience and to formal mathematical definition, optimal intensity of the embodied-based activity to support learning in mathematics, and differentiation in understanding between students who learn through an embodied-based activity and who learn through a normal approach. Additionally, the statistical techniques are highly recommended as quantitative study of embodied-based learning can be achieved. In quantitative dimension, students' achievement in various types of comparison, level of understanding and concept mapping can be quantified based on theoretical supports.

In summary, through the embodiment, conceptual metaphor and blending occurred in students and led to proper image, proper reasons, hence, proper concept of infinite sets comparison. Prior to the blend, image having or metaphor is an essential step as ones learn how taking away paired elements relate to equivalent sets. Then the conceptual blending will blend their embodied-based experience and their constructed knowledge to the formal definition. However, students should start their learning journey from having experiences that allow the embodiment to be meaningful and relevant to desired mathematical concepts (Tall, 2008). We can think of an embodied-based activity as an alternative approach that teachers can follow to create some meaningful experience, influencing the mitigation of learning difficulties. Teachers can start with tangible manipulatives that represent small finite sets with graphical and numerical representations, then extend to large sets and finally go forward to infinite sets. Sequential tasks of EBIC could be the model to development of instructional approach concerning about cognitive foundation before complicated conceptualization. Taking this process as an advantage, multimodal curriculum designers can apply embodiedbased experience to help learners to conceptualize, facilitate them by providing this tangible, easily perceivable and meaningful experience. Hence, learning mathematics can be more accessible for lower-ability students and even disability persons, such as a visually impaired person (Sedaghatjou, 2017).

\section{ACKNOWLEDGEMENT}

This research was partially supported by the Thailand Research Fund (TRG5880196) and The Institute for the Promotion of Teaching Science and Technology (IPST). The authors would like to thank Ms.Suparat Chuechote for constructive criticism and proofreading the manuscript.

\section{REFERENCES}


Abrahamson, D., Trninic, D., Gutiérrez, J. F., Huth, J., \& Lee, R. G. (2011). Hooks and shifts: A dialectical study of mediated discovery. Technology, Knowledge, and Learning, 16(1), 55-85.

Abrahamson, D., \& Lindgren, R. (2014). Embodiment and embodied design. In R. K. Sawyer (Eds), The Cambridge handbook of the learning sciences $\left(2^{\text {nd }}\right.$ ed.) (pp. 358376). Cambridge, UK: Cambridge University Press.

Alibali, M. W., \& Nathan, M. J. (2012). Embodiment in mathematics teaching and learning: Evidence from learners' and teachers' gestures. Journal of the Learning Sciences, 21, 247-286.

Arzarello, F., Paola, D., Robutti, O., \& Sabena, C. (2009). Gestures as semiotic resources in the mathematics classroom. Educational Studies in Mathematics, 70, 97109.

Barsalou, L. W. (1999). Perceptual symbol systems. Behavioral and Brain Science, 22, 577-660.

Chahine, I. C. (2013). The impact of using multiple modalities on students' acquisition of fractional knowledge: An international study in embodied mathematics across semiotic cultures. Journal of Mathematical Behavior, 32(3), 434-449.

Denbel, D. G. (2014). Students' misconceptions of the limit concept in a first calculus course. Journal of Education and Practice, 5(34), 24-41.

Denzin, N. K., \& Lincoln, Y. S. (2005). The sage handbook of qualitative research. London: Sage Publications.

Edwards, L. D., \& Robutti, O. (2014). Embodiment, modalities, and mathematical affordances. In L. D. Edwards, F. Ferrara, \& D. Moore-Russo (Eds.), Emerging perspectives on gesture and embodiment in mathematics (pp.1-23). Charlotte, NC: Information Age Publishing.

Fischbein, E., Tirosh, D., \& Hess, P. (1979). The intuition of infinity. Educational Studies in Mathematics, 10, 3-40.

Kul, Ü., Çelik, S., \& Aksu, Z. (2018). The impact of educational material use on mathematics achievement: A meta-analysis. International Journal of Instruction, 11(4), 303-324.

Lakoff, L., \& Núñez, R. (2000). Where mathematics come from: How the embodied mind brings mathematics into being. New York: Basic Books.

Mamolo, A., \& Zazkis, R. (2008). Paradoxes as a window to infinity. Research in Mathematics Education, 10(2), 167-182.

Manches, A., O’Malley, C., \& Benford, S. (2010). The role of physical representations in solving number problems: A comparison of young children's use of physical and virtual materials. Computers and Education, 54(3), 622-640. 
Nathan, M. J., \& Walkington C. (2017). Grounded and embodied mathematical cognition: Promoting mathematical insight and proof using action and language. Cognitive Research: Principles and Implications, 2(1), 2-20. doi: 10.1186/s41235-0160040-5.

Núñez, R. E. (2005). Creating mathematical infinities: Metaphor, blending, and the beauty of transfinite cardinals. Journal of Pragmatics, 37, 1717-1741.

Parsley, J., \& Soriano, C., T. (2009). Understanding geometry in the dance studio. Journal of Mathematics and the Arts, 3(1), 11-18.

Petrick, C., King, B., \& Hoyte, J. (2014). The journal of mathematical behavior learning angles through movement: Critical actions for developing understanding in an embodied activity. Journal of Mathematical Behavior, 36, 95-108.

Rizzolatti, G., Fagiga, L., \& Fogassi, L. (1996). Premotor cortex and the recognition of motor actions. Cognitive Brain Research, 3, 131-141.

Singer, F. M., \& Voica, C. (2008). Between perception and intuition: Learning about infinity. Journal of Mathematical Behavior, 27(3), 188-205.

Sedaghatjou, M. (2017). Advanced mathematics communication beyond modality of sight. International Journal of Mathematical Education in Science and Technology, 49(1), 46-65.

Tall, D. (2008). The transition to formal thinking in mathematics. Mathematics Education Research Journal, 20(2), 5-24.

Tirosh D. (2002) The role of students' intuitions of infinity in teaching the Cantorian theory. In D. Tall (Eds), Advanced mathematical thinking (pp. 199-241). Mathematics Education Library, vol 11. Springer, Dordrecht.

Tsamir, P. (1999). Prospective teachers' acceptance of one-to-one correspondence criterion for comparing infinite sets. In O. Zaslavsky (Eds), Proceedings of the 23rd Conference of the International Group for the Psychology of Mathematics Education (Vol. 4) (pp. 305-312). Israel: PME.

Tsamir, P., \& Tirosh, D. (1999). Consistency and representations: The case of actual infinity. Journal for Research in Mathematics Education, 30, 213-219.

Tsamir, P., \& Dreyfus, T. (2002). Comparing infinite sets-A process of abstraction: The case of Ben. Journal of Mathematical Behavior, 21, 1-23.

Tsamir, P., \& Tirosh, D. (2007). Teaching for conceptual change: The case of infinite sets. In S. Vosniadou, A. Baltas, \& X. Vamvakoussi (Eds.), Reframing the conceptual change approach in learning and instruction (pp. 299-316). Oxford, UK: Elsevier.

Wijeratne, C., \& Zazkis, R. (2015). On painter's paradox: Contextual and mathematical approaches to infinity. International Journal of Research in Undergraduate Mathematics Education, 1(1), 163-186. 